RODRIGUES, S. M. S.; TELES, A. C.; RIBEIRO, M. J. B.; SANTOS, M. J. C. A relevância do curso de Ciência da Computação para a inovação e desenvolvimento da ciência e tecnologia

\title{
A RELEVÂNCIA DO CURSO CIÊNCIA DA COMPUTAÇÃO PARA A INOVAÇÃO E DESENVOLVIMENTO DA CIÊNCIA E TECNOLOGIA
}

\author{
Simone Maria da Silva Rodrigues \\ Mestranda no Curso Ciência da Propriedade Intelectual na Universidade Federal de Sergipe-UFS \\ e-mail: smsr.direito@hotmail.com \\ Ângela das Chagas Teles \\ Mestranda no Curso Ciência da Propriedade Intelectual na UFS \\ e-mail: angelita.teles@ hotmail.com \\ Marta Jeidjane Borges Ribeiro \\ Doutoranda no Curso Ciência da Propriedade Intelectual na UFS \\ e-mail: emestatistica@gmail.com \\ Mário Jorge Campos dos Santos \\ Professor Pós-doutor no Programa de Pós-graduação em Ciência da Propriedade Intelectual na UFS \\ e-mail:mjkampos@gmail.com
}

Recebido em: 01/10/2015

Aprovado em: 07/12/2015

\begin{abstract}
RESUMO
O avanço tecnológico e as crescentes mudanças que o mundo e a sociedade vêm enfrentando ao longo dos anos, nos demonstra que são essenciais profissionais capacitados para criar, manusear e auxiliar as inovações que são lançadas no mercado consumidor. Dessa forma, o objetivo desse estudo foi demonstrar a relevância do curso Ciência da Computação para inovação e desenvolvimento da ciência e tecnologia, e verificar o cenário quantitativo dos candidatos que concorreram a uma vaga do respectivo curso nos vestibulares da Universidade Federal de Sergipe (UFS) no período de (2002 a 2013). Com o resultado da pesquisa, percebeu-se que em 2010 houve mais inscritos no curso $(423 ; 406)$, a maioria dos inscritos e classificados foram do sexo masculino, somente apenas a partir de $2010 \mathrm{em}$ diante, observouse um número crescente de inscritos e classificados do sexo feminino. Isso posto, constatou-se que o curso Ciência da Computação é extremamente relevante e contribui para inovação e desenvolvimento da ciência e tecnologia.
\end{abstract}

Palavras-chave: Ciência da Computação. Inovação. Universidade Federal de Sergipe.

\section{THE RELEVANCE OF COMPUTER SCIENCE COURSE FOR INNOVATION AND TECHNOLOGY SCIENCE DEVELOPMENT}


RODRIGUES, S. M. S.; TELES, A. C.; RIBEIRO, M. J. B.; SANTOS, M. J. C. A relevância do curso de Ciência da Computação para a inovação e desenvolvimento da ciência e tecnologia

\begin{abstract}
Technological advances and the increasing changes that the world and society have faced over the years shows that trained professionals are essential to create, handle and assist the innovations that are launched in the consumer market. Thus the aim of this study was to demonstrate the relevance of computer science course for innovation and development of science and technology, and check the quantitative scenario of candidates who competed for a spot of their course in the entrance of the Federal University of Sergipe (UFS) in period (2002-2013). With the search result, it was noted that in 2010 and in 2010 there were more enrolled in the course $(423 ; 406)$, the majority of subscribers and classified were male, only from 2010 onwards, there was a number increasing subscribers and classified female students. According to this, it was found that the Computer Science course is extremely important and contributes to innovation and development of science and technology.
\end{abstract}

Keywords: Computer Science. Innovation. Federal University of Sergipe.

\title{
1 INTRODUÇÃO
}

Com o avanço tecnológico e as crescentes mudanças que o mundo e a sociedade vêm enfrentando ao longo dos anos, é essencial termos profissionais capacitados para criar, manusear e auxiliar as inovações que são lançadas no mercado consumidor. Com isso, diversos campos, desafios e oportunidades vão surgindo para aqueles que lidam com ciência e tecnologia e querem ingressar no mercado de trabalho relacionado com ciência da computação.

Nessa lógica, o Instituto Federal de Minas Gerais, campus Formiga (2015, p. 1) diz que:

\begin{abstract}
A evolução tecnológica e as transformações sociais e econômicas exigem que as Escolas reformulem o seu papel como Centro de Formação Profissional de forma a atender às demandas do mundo do trabalho. Tem-se observado a exigência de competitividade no sentido de se obter produtos e serviços com qualidade e produtividade. O setor de informática tem sido um dos fatores de dinamização do funcionamento das empresas de todas as áreas produtivas. Não se pode conceber nos tempos atuais a produção agrícola, industrial e de comércio e serviços e nem a própria vida das pessoas sem a informática. Assim, o evidente crescimento da área exige a qualificação das pessoas em todos os níveis, reforçando a iniciativa da Escola em formar profissionais empreendedores, capazes de atender às expectativas do setor em nível local e regional.
\end{abstract}


RODRIGUES, S. M. S.; TELES, A. C.; RIBEIRO, M. J. B.; SANTOS, M. J. C. A relevância do curso de Ciência da Computação para a inovação e desenvolvimento da ciência e tecnologia

De acordo com estudo realizado pelo Ministério da Ciência e Tecnologia junto a Secretaria de Política de Informática (2009/2010, p. 11), aponta-se que

\footnotetext{
a qualificação dos recursos humanos empregados pelas Instituições de Ciência e Tecnologia (ICTs) das regiões NE/CO, observou que o emprego de profissionais de nível superior na área de P\&D das instituições aumentou em sete vezes (102 para 675), o que demonstra uma variação significativa, principalmente, quando comparada ao mesmo indicador na região Sudeste, onde o número apenas dobrou (869 para 1859). Porém, observou-se que a base de profissionais nas regiões NE/CO era pequena e o crescimento do volume de recursos, como comentado, foi acentuado.
}

Com isso, percebe-se que o campo da ciência, tecnologia e inovação é diretamente ligado à pesquisa e desenvolvimento, e, consequentemente, ao curso Ciência da Computação, pois através desse curso, temos profissionais aptos a identificar problemas do mundo real, propor soluções inéditas ou melhorar as já existentes, tornando-as operantes no que diz respeito à efetivação de um determinado projeto, por meio da construção de modelos computacionais e de sua implementação (FACULDADE ALVORADA, 2015).

Cunha (2007, p. 1), diz que as áreas que mais cresceram no país na comparação dos triênios 2001-2003 e 2004-2006, foram psicologia e psiquiatria (70\%); produção animal e vegetal (58\%); ciências sociais (52\%); medicina (47\%); farmacologia (46\%); ciência agronômica (46\%); imunologia (44\%); computação (44\%)[...].

Nesse sentido, o objetivo desse estudo é demonstrar a relevância do curso Ciência da Computação para inovação e desenvolvimento da ciência e tecnologia, e verificar o cenário quantitativo dos candidatos que concorreram a uma vaga do respectivo curso nos vestibulares da Universidade Federal de Sergipe (UFS) no período de 2002 a 2013.

\section{REFERENCIAL TEÓRICO}

De acordo com Tigre (1999, p. 98), “o Brasil se destaca pelo elevado número absoluto de pessoas (aproximadamente $550 \mathrm{mil}$ ) atuando em informática, tanto como usuários profissionais quanto em atividades de suporte".

Maldonado (1999, p. 105) relata que "o acesso a uma ampla base de informações e conhecimentos científicos e tecnológicos, que se constituía numa vantagem no passado, tornou-se uma necessidade fundamental no presente".

Para Luryi et al (2007, p. 1): 
RODRIGUES, S. M. S.; TELES, A. C.; RIBEIRO, M. J. B.; SANTOS, M. J. C. A relevância do curso de Ciência da Computação para a inovação e desenvolvimento da ciência e tecnologia

\begin{abstract}
As grandes e recentes mudanças no mundo apresentam desafios e oportunidades para o ensino da engenharia. $\mathrm{O}$ ambiente que enfrentamos agora é muito diferente do que foi há uma década atrás. A prática da engenharia está mudando com a globalização na produção, pesquisa e desenvolvimento. Novos campos interdisciplinares surgiram e a engenharia tradicional é cada vez mais influenciada pela tecnologia da informação. Mudanças para melhor na atividade empreendedora das empresas frequentemente localizadas no mar.
\end{abstract}

Nessa perspectiva percebemos claramente que os autores, destacam o quantitativo de profissionais e suporte na área de informática e as crescentes mudanças com os avanços da ciência e tecnologia no mundo atual, pois o cenário do passado não é o que temos no presente. As empresas, as inovações e a globalização trouxeram a necessidade de se investir em tecnologia e inovação e, em decorrência desse cenário, verifica-se a necessidade de haver profissionais capacitados para atender o mercado e contribuir com essas mudanças.

Nesse diapasão Maldonado (1999, p. 105) relata que:

\begin{abstract}
Assiste-se a um acirramento da concorrência entre os agentes econômicos, ao mesmo tempo em que o conhecimento científico e tecnológico vem ocupando um papel absolutamente central nas suas estratégias competitivas. Uma vez que um crescente número de empresas se vem confrontando com novas trajetórias tecnológicas, pelo advento das novas tecnologias, a necessidade de informação sobre futuros desenvolvimentos torna-se ainda mais vital.
\end{abstract}

Na mesma linha de raciocínio Álvaro (2012, p. 4), diz que: "nos últimos anos, a inovação científica e tecnológica tem se estabelecido como um dos fatores mais importantes para garantir crescimento, competitividade e rentabilidade diferenciada às empresas".

Diante disso, verificou-se que ciência, tecnologia e inovação, estão diretamente ligadas ao curso Ciência da Computação, pois o curso forma e habilita o profissional na área, que é fundamental no processo de desenvolvimento, competitividade, evolução e manutenção dos serviços ligados à tecnologia das empresas em todos os ramos de atividade. $\mathrm{Na}$ atualidade, dificilmente uma instituição ou pessoa conduz suas atividades sem a ciência da computação.

\title{
3 CURSO DE CIÊNCIA DA COMPUTAÇÃO NA UNIVERSIDADE FEDERAL DE SERGIPE (UFS)
}


RODRIGUES, S. M. S.; TELES, A. C.; RIBEIRO, M. J. B.; SANTOS, M. J. C. A relevância do curso de Ciência da Computação para a inovação e desenvolvimento da ciência e tecnologia

De acordo com catálogo de cursos graduação da Universidade Federal de Sergipe UFS (2015, p. 33), o curso Ciência da Computação tem duração de 4 anos, oferta 100 vagas anuais, se localiza no campus São Cristóvão, funciona no turno diurno, e o acadêmico, no final, recebe o título de Bacharel em Ciência da Computação.

Seu projeto pedagógico é regulamentado pela Resolução 49/2008 do Conselho do Ensino, da Pesquisa e da Extensão (CONEPE). Conforme seu artigo $2^{\circ}$ o Curso de Graduação em Ciência da Computação tem como objetivo geral graduar profissionais para atuarem no desenvolvimento científico e tecnológico da computação, através do uso do computador no processamento de informações para fins administrativos e técnicos, com visão humanística crítica perante problemas sociais relativos à profissão e capazes de assumir responsabilidades sociais e políticas.

Como perfil, o Bacharel em Ciência da Computação deverá assumir um papel de agente transformador do mundo do trabalho, apto a provocar mudanças através do desenvolvimento e incorporação de novas tecnologias da informação na solução de problemas organizacionais e possui domínio sobre:

I. racionalização e automação de rotinas administrativas das organizações, e no suporte aos seus sistemas de controle operacional e gerencial, através da análise, projeto e implementação de sistemas;

II. aplicações técnicas ou científicas que envolvem modelos matemáticos capazes de serem resolvidos numericamente pelo computador, e,

III. construção de software e de outros projetos complexos no processamento das informações.

Em relação às competências e habilidades a serem adquiridas pelo Bacharel em Ciência da Computação, ao longo do desenvolvimento das atividades curriculares e complementares do curso, de acordo com artigo $4^{\circ}$ da Resolução no 49/2008 CONEPE são, dentre outras:

I. desenvolver trabalhos na área de racionalização e automação de rotinas administrativas das organizações, e no suporte aos seus sistemas de controle operacional e gerencial, através da análise, projeto e implementação de sistemas;

II. desenvolver trabalhos em aplicações técnicas ou científicas que envolvem modelos matemáticos capazes de serem resolvidos numericamente pelo computador; III. desenvolver projetos de construção de software e de outros projetos complexos no processamento das informações;

IV. selecionar software ou hardware adequados às necessidades empresariais, administrativas de ensino e de pesquisa;

V. atualizar-se constantemente, visando a acompanhar o estado da arte; 
RODRIGUES, S. M. S.; TELES, A. C.; RIBEIRO, M. J. B.; SANTOS, M. J. C. A relevância do curso de Ciência da Computação para a inovação e desenvolvimento da ciência e tecnologia

VI. desenvolver trabalhos em grupo e de disseminar o conhecimento na área de computação;

VII. dedicar-se a atividades de pesquisa científica;

VIII. empreender no setor de informática, e,

IX. possuir visão humanística crítica e consistente com sua atuação profissional e respeitar os princípios éticos da área de computação.

O Curso de Graduação em Ciência da Computação, modalidade Bacharelado será ministrado com a carga horária mínima de 3510 (três mil quinhentas e dez) horas, que equivalem a 234 (duzentos e trinta e quatro) créditos, sendo 182 (cento e oitenta e dois) créditos obrigatórios e 52 (cinquenta e dois) créditos optativos/atividades complementares, conforme definido no Projeto Pedagógico do Curso (Res.49/2008 CONEPE).

\section{METODOLOGIA}

Para a realização deste estudo, utilizou-se a metodologia de pesquisa bibliográfica, tipo descritiva e quantitativa. $\mathrm{O}$ mapeamento foi realizado tendo como base os anuários da Universidade Federal de Sergipe disponibilizados no site da instituição. O foco do estudo foi demonstrar a importância do curso Ciência da Computação para o desenvolvimento da ciência tecnologia e inovação, e verificar o cenário quantitativo dos candidatos que concorreram a uma vaga do respectivo curso nos vestibulares da Universidade Federal de Sergipe (UFS) no período de (2002 a 2013). O levantamento foi realizado em julho de 2015.

Os dados encontrados foram computados individualmente e organizados em planilha para elaboração dos gráficos e tabelas, por meio do Microsoft Office Excel, a fim de caracterizar o quantitativo dos inscritos no vestibular da Universidade Federal de Sergipe, que optaram pelo curso Ciência da Computação, considerando o ano do vestibular, número de inscritos, número de vagas ofertadas, número de concorrentes, classificados e contabilizados por sexo.

\section{RESULTADOS E ANÁLISE}

Analisou-se por ano do vestibular as vagas ofertadas, número de inscritos e concorrência por vaga no curso Ciência da Computação da UFS (TAB. 1). Verificou-se que 
RODRIGUES, S. M. S.; TELES, A. C.; RIBEIRO, M. J. B.; SANTOS, M. J. C. A relevância do curso de Ciência da Computação para a inovação e desenvolvimento da ciência e tecnologia

de 2002 a 2004 eram ofertadas apenas 30 vagas, em 2005 até 2008 passou para (50), a partir de 2009 até 2013 foi duplicada a quantidade de vagas ofertadas passando para (100) no total.

Verificou-se que o número de inscritos variou conforme os anos com maior incidência no ano de 2010, o qual atingiu o total de 423 candidatos. O menor número foi em 2007 com o total de 206 candidatos; Mesmo com o menor quantitativo de candidatos, esse não foi o ano que obteve menor concorrência, tendo em vista que, nesse período foram ofertadas apenas 50 vagas, a concorrência foi de 4,12 por vaga (TAB. 1).

Constatou-se que houve o maior número de concorrentes em 2002 (11,47 por vaga), 2003 (13,33 por vaga) e 2004 (12,9 por vaga), porém, observou-se que nesses anos a quantidade de vagas oferecidas eram menores (30), o que de fato, influenciou no número de concorrentes por vaga. O ano que obteve a menor concorrência foi o de 2009, no qual candidataram-se 298 pessoas, e foram ofertadas 100 vagas, chegando a atingir a concorrência de 2,98 por vaga (TAB. 1 ).

Tabela 1 - Resumo dos vestibulares: curso Ciência da Computação na UFS, 2002-2013

\begin{tabular}{c|c|c|c}
\hline Ano & Vagas & Inscritos & $\begin{array}{c}\text { Concorrência } \\
\text { por vaga }\end{array}$ \\
\hline 2002 & 30 & 344 & 11,47 \\
\hline 2003 & 30 & 400 & 13,33 \\
\hline 2004 & 30 & 387 & 12,9 \\
\hline 2005 & 40 & 325 & 8,13 \\
\hline 2006 & 50 & 378 & 7,56 \\
\hline 2007 & 50 & 206 & 4,12 \\
\hline 2008 & 50 & 370 & 7,4 \\
\hline 2009 & 100 & 298 & 2,98 \\
\hline 2010 & 100 & 423 & 4,23 \\
\hline 2011 & 100 & 335 & 3,35 \\
\hline 2012 & 100 & 324 & 3,24 \\
\hline 2013 & 100 & 406 & 4,06 \\
\hline
\end{tabular}

Fonte: Anuários da UFS (2004 a 2014).

Quanto ao número de inscritos nos vestibulares de 2002 a 2013 que optaram a concorrer uma vaga do Curso Ciência da Computação (FIG. 1), constatou-se que houve 
RODRIGUES, S. M. S.; TELES, A. C.; RIBEIRO, M. J. B.; SANTOS, M. J. C. A relevância do curso de Ciência da Computação para a inovação e desenvolvimento da ciência e tecnologia

oscilação no número de inscritos, porém, a maioria permaneceu entre 300 a 400 candidatos, como observado nos anos 2002; 2003; 2004; 2005; 2006; 2008; 2011; 2012. O ano que apresentou o menor número de candidatos foi o de 2007 com o total de 206 candidatos, o maior número de inscritos foi em 2010 e em 2013, com 423 e 406 inscritos, respectivamente.

Figura 1 - Número de inscritos nos vestibulares da UFS concorrentes à vaga do Curso Ciência da Computação no período de 2002 a 2013

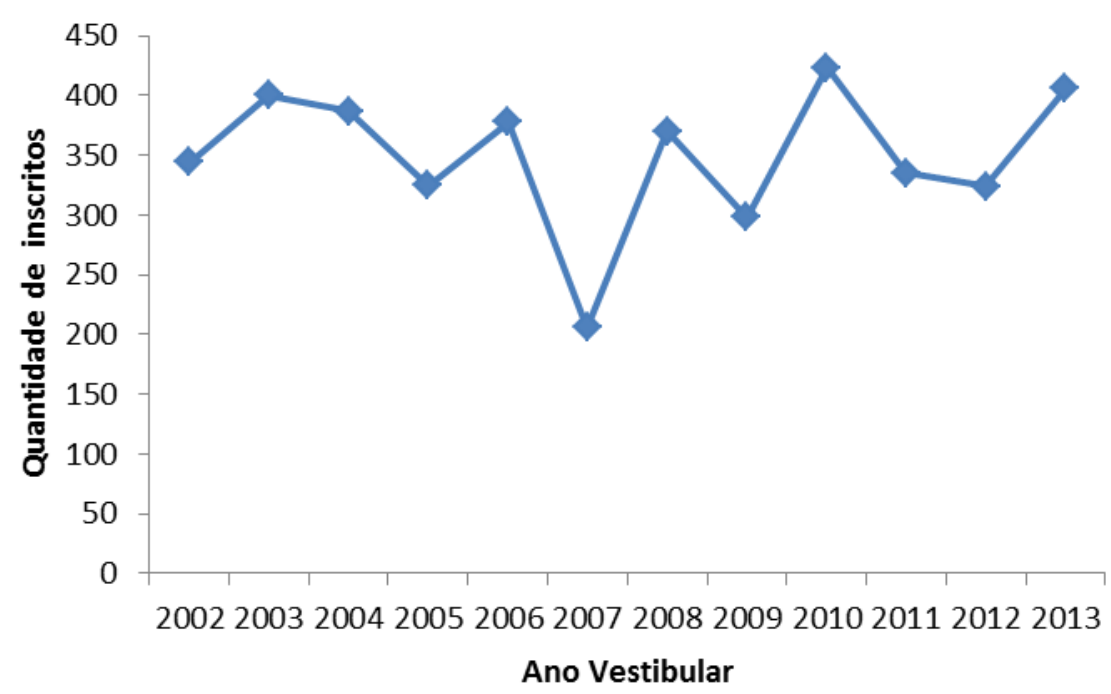

Fonte: Anuários da UFS (2004 a 2014).

Referente à quantidade de candidatos inscritos nos vestibulares de 2002 a 2013, que optaram pelo Curso Ciência da Computação, porém, divididos por sexo (FIG. 2), observou-se que a maioria dos inscritos são do sexo masculino, que confirma o que foi dito por Pricilla Honorato em 03 de junho de 2015: "Em 2012, apenas 14\% das jovens brasileiras que entraram na universidade pela primeira vez escolheram campos relacionados à ciência, incluindo engenharia, indústria e construção. Em contrapartida, 39\% dos jovens do sexo masculino que entraram na universidade naquele ano optaram por seguir uma dessas áreas. É o que revela um estudo da Organização para a Cooperação e Desenvolvimento Econômico (OCDE)".

Constatou-se que os anos 2002; 2003; 2004; 2006; 2010 apresentaram os maiores números de candidatas do sexo feminino, destacando-se o ano de 2010 com 82 candidatas inscritas (FIG. 2). 
RODRIGUES, S. M. S.; TELES, A. C.; RIBEIRO, M. J. B.; SANTOS, M. J. C. A relevância do curso de Ciência da Computação para a inovação e desenvolvimento da ciência e tecnologia

Figura 2 - Quantidade de inscritos nos vestibulares (2002 a 2013) da UFS que optaram pelo Curso Ciência da Computação, divididos por sexo

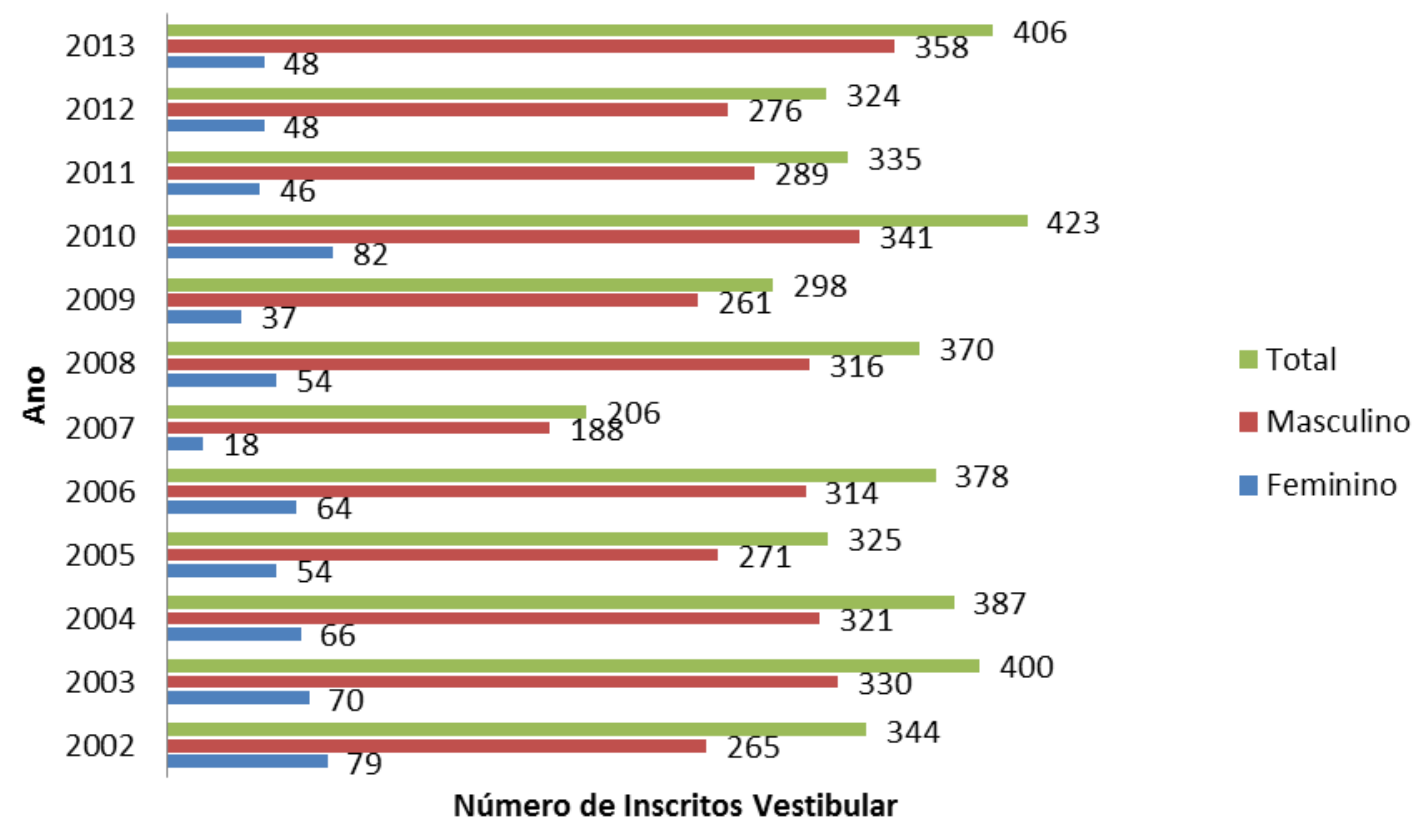

Fonte: Anuários da UFS (2004 a 2014).

Ao analisar a quantidade de inscritos classificados nos vestibulares divididos por sexo (FIG. 3), constatou-se que a maioria dos classificados nos vestibulares de 2002 a 2013 que optaram pelo curso Ciência da Computação da UFS foram do sexo masculino, o que se justifica pelo fato do número de pessoas inscritas também serem a maioria do sexo masculino. Observou-se que a partir dos anos de 2010; 2011; 2012 e 2013 houve um aumento no número de classificados do sexo feminino, se estabilizando entre 12 a 15 candidatas classificadas. $\mathrm{O}$ ano com menor classificação foi em 2004 no qual, das 30 vagas ofertadas, apenas 1 candidato classificado foi do sexo feminino. 
RODRIGUES, S. M. S.; TELES, A. C.; RIBEIRO, M. J. B.; SANTOS, M. J. C. A relevância do curso de Ciência da Computação para a inovação e desenvolvimento da ciência e tecnologia

Figura 3 - Quantidade de classificados nos vestibulares 2002 a 2013 da UFS que optaram pelo Curso Ciência da Computação, divididos por sexo

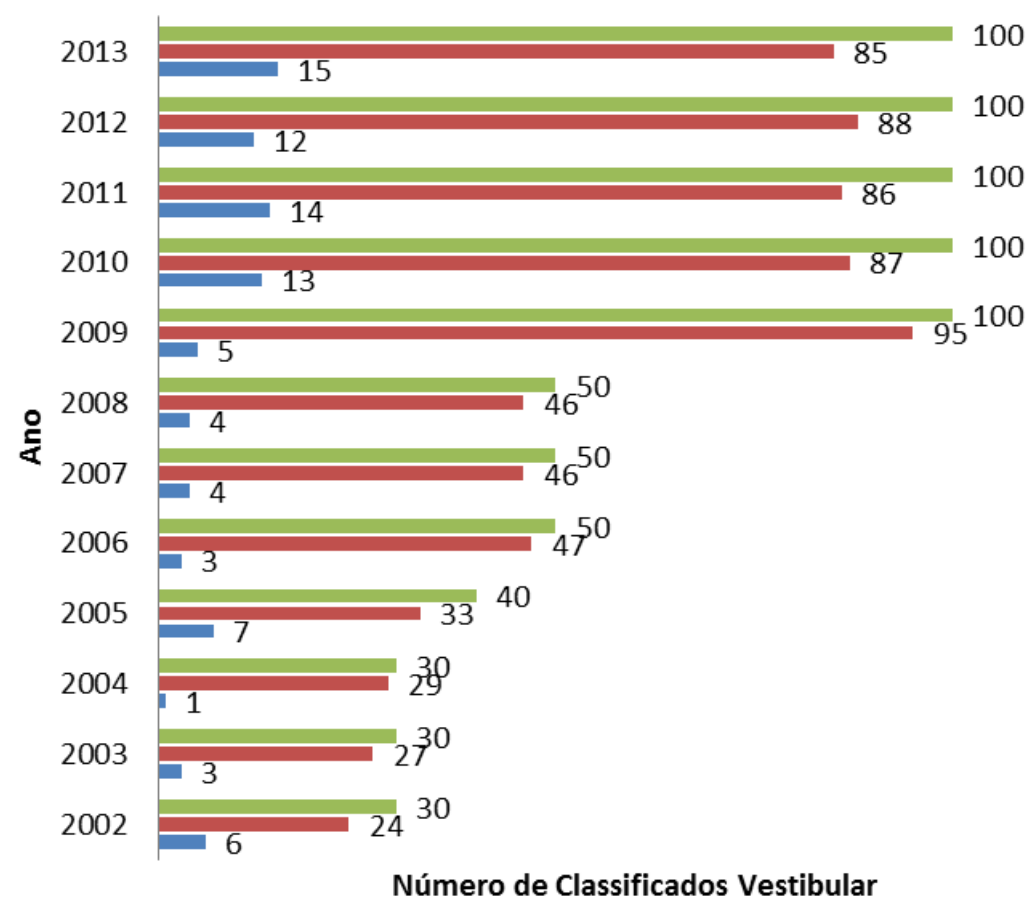

Vagas ofertadas

Masculino

Feminino

Fonte: Anuários da UFS (2004 a 2014)

\section{CONCLUSÃO}

Após as explanações descritas, verificou-se que o curso Ciência da Computação está diretamente ligado à inovação, ciência e tecnologia. Gradua profissionais essenciais para a manutenção, criação e desenvolvimento científico e tecnológico das empresas e instituições ligadas à atividade.

Constatou-se que a Universidade Federal de Sergipe, oferece o curso com 4 anos de duração, é localizado no campus São Cristóvão, oferta 100 vagas anuais, funciona no turno diurno, e, o acadêmico, ao final da graduação, recebe o título de Bacharel em Ciência da Computação.

Referente às vagas ofertadas para o curso no período de 2002 a 2013, observou-se que praticamente o número triplicou, subiu de 30 vagas anuais para 100 no total, representando mais de $300 \%$ de aumento no número de vagas oferecidas pela instituição. 
RODRIGUES, S. M. S.; TELES, A. C.; RIBEIRO, M. J. B.; SANTOS, M. J. C. A relevância do curso de Ciência da Computação para a inovação e desenvolvimento da ciência e tecnologia

Em relação ao número de inscritos nos vestibulares de 2002 até 2013, os anos que apresentaram o maior número de inscritos foram o de 2010 com 423 e o de 2013 com 406. Quanto à quantidade de inscritos divididos por sexo, certificou-se que a maioria dos inscritos foi do sexo masculino, e o ano que obteve o maior número de inscritos do sexo feminino foi o de 2010 com 82 candidatas inscritas. No tocante à classificação dividida por sexo, detectou-se que a maioria dos classificados foi do sexo masculino, somente a partir de 2010, em seguida, o número de classificados do sexo feminino aumentou, se estabilizando entre 12 a 15 classificadas.

Portanto, o curso de Ciência da Computação é fundamental no processo tecnológico e inovador, e a Universidade Federal de Sergipe está contribuindo com esse desenvolvimento capacitando pessoas e aumentado o número de vagas ofertadas para o curso, ao longo dos anos.

\section{REFERÊNCIAS}

ÁLVARO, A. Empreendedorismo e inovação em tecnologia da informação e comunicação (TIC) no curso de bacharelado em Ciência da Computação. In: CONGRESSO DA SOCIEDADE BRASILEIRA DE COMPUTAÇÃO, 32., 2012, Curitiba. Anais... Curitiba, SBC, 2012. Disponível em: <http://www.imago.ufpr.br/csbc2012/anais_csbc/eventos/ wei/artigos/Empreendedorismo\%20e\%20Inovacao\%20em\%20Tecnologia\%20da\%20Informa cao $\% 20 \mathrm{e} \% 20$ Comunicacao $\% 20(\% 20 \mathrm{TIC} \% 20) \% 20$ no $\% 20$ Curso $\% 20 \mathrm{de} \% 20$ Bacharelado $\% 20 \mathrm{e}$ m\%20Ciencia\%20da\%20Computacao.pdf>. Acesso em: 21 jul. 2015.

CONEPE-Conselho do Ensino, da Pesquisa e da Extensão. Resolução n49/2008. Disponível em: <https://www.sigrh.ufs.br/sigrh/public/colegiados/filtro_busca.jsf;jsessionid=052376673 256C8BC74AA43F5D205F140.carauna1>. Acesso em: 22 jul. 2015.

CUNHA, A. Produção científica: Brasil sobe no ranking. Belém: MEC, 2007.

Disponível em: <http://portal.mec.gov.br/index.php?option=com_content\&view=article\&id= 8576:\&catid=180\&Itemid=164>. Acesso em: 26 jul. 2015.

FACULDADE ALVORADA. Ciência da Computação. Disponível em:

<http://alvoradamaringa.com.br/curso-ciencia-da-computacao.php>. Acesso em: 20 jul. 2015.

HONORATO, P. Participação de mulheres em carreiras de exatas e científicas é baixa.

Observatório do PNE. 2015. Disponível em: <http://www.observatoriodopne.org.br/ noticias/participacao-de-mulheres-em-carreiras-de-exatas-e-cientificas-e-baixa>. Acesso em: 22 jul. 2015. 
RODRIGUES, S. M. S.; TELES, A. C.; RIBEIRO, M. J. B.; SANTOS, M. J. C. A relevância do curso de Ciência da Computação para a inovação e desenvolvimento da ciência e tecnologia

IFMG- Instituto Federal de Minas Gerais, Campus Formiga. Ciência da Computação. Disponível em: <http://formiga.ifmg.edu.br/index.php?option=comcontent\&view $=$ article\&id=405\&Itemid=134>. Acesso em: 26 jul. 2015.

LURYI, S. et al. Entrepreneurship in Engineering Education. In: ANNUAL FRONTIERS IN EDUCATION CONFERENCE, 37., 2007. Proceedings... USA, 2007. Disponível em: <http://fie-conference.org/fie2007/papers/1705.pdf>. Acesso em: 26 jul. 2015.

MALDONADO, J. Tecno-globalismo e acesso ao conhecimento. In: LASTRES, H.;

ALBAGLI, S. (Org.). Informação e globalização na era do conhecimento. Rio de Janeiro: UFF, 1999. p. 105-121.

MINISTÉRIO DA CIÊNCIA E TECNOLOGIA-MCT. Secretaria de Política de Informática. Projeto avaliação da política de informática. Brasília, mar. 2009/dez. 2010.

Disponível em: <www.mct.gov.br/upd_blob/0219/219360.pdf>. Acesso em: 22 jul. 2015.

TIGRE, P. B. Comércio eletrônico e globalização: desafios para o Brasil. In: LASTRES, H.; ALBAGLI, S. (Org.). Informação e globalização na era do conhecimento. Rio de Janeiro: UFF, 1999. p. 84-104.

UNIVERSIDADE FEDERAL DE SERGIPE-UFS. Anuário estatístico. Disponível em: <http://oficiais.ufs.br/pagina/anu-rio-estat-stico-5839.html>. Acesso em: 22 jul. 2015. 